\title{
An adaptive phase space method with application to reflection traveltime tomography
}

\author{
Eric Chung* Jianliang Qian ${ }^{\dagger} \quad$ Gunther Uhlmann ${ }^{\ddagger} \quad$ Hongkai Zhao $^{\S}$
}

November 25, 2010

\begin{abstract}
In this work an adaptive strategy for the phase space method [5] for traveltime tomography is developed. The method first uses those geodesics/rays that produce smaller mismatch with the measurements and continues on in the spirit of layer stripping without defining the layers explicitly. The adaptive approach improves stability, efficiency and accuracy. We then extend our method to reflection traveltime tomography by incorporating broken geodesics/rays, for which a jump condition has to be imposed at the broken point for the geodesic flow. In particular we show that our method can distinguish non-broken and broken geodesics in the measurement and utilize them accordingly in reflection traveltime tomography. We demonstrate that our method can recover the convex hull (with respect to the underlying metric) of unknown obstacles as well as the metric outside the convex hull.
\end{abstract}

\section{Introduction}

Traveltime tomography deals with the problem of determining the internal properties of a medium by measuring the traveltimes of waves going through the medium. It arises in global seismology in determining the inner structure of Earth by measuring at different seismic stations the traveltimes of seismic waves produced by earthquakes. It also arises in exploration geophysics, in particular, hydrocarbon exploration. For instance in marine reflection seismology the data is collected on a ship with a streamer that sends out sound waves and receives the response on hydrophones or receiver groups.

Traveltime tomography also arises in medical imaging, in particular, in ultrasound computed tomography (UTT). In UTT the acoustic speed in biological tissues can be calculated from the arrival times of ultrasonic waves. Another area of application of traveltime tomography is ocean acoustics. For sound waves travelling horizontally in the ocean, speed is largely function of temperature. Thus the traveltime of a wave of sound between two points is a sensitive indicator of the temperature along its path.

\footnotetext{
*Department of Mathematics, The Chinese University of Hong Kong, Hong Kong. Email: tschung@math.cuhk.edu.hk

${ }^{\dagger}$ Department of Mathematics, Michigan State University, East Lansing, MI 48824. Email: qian@math.msu.edu

${ }^{\ddagger}$ Department of Mathematics, University of California, Irvine, CA92697-3875. Email: guhlmann@math.uci.edu

${ }_{\S}^{\S}$ Department of Mathematics, University of California, Irvine, CA92697-3875. Email: zhao@math.uci.edu
} 
Recent progress in boundary rigidity and lens rigidity problems in Riemannian geometry [43, $44,46,48,45,50,51,52,30,31,15,21]$ has motivated us to transfer these theoretical advances into numerical algorithms for recovering a Riemannian manifold; see [5, 6, 22, 23] for such algorithmic developments. In $[5,6]$ we have developed phase space methods for recovering such Riemannian manifolds in terms of index of refraction in acoustic and isotropic elastic media from transmission traveltimes. In this paper, we incorporate an adaptive strategy into the phase space method and apply the resulting adaptive method to recovering index of refraction from reflection traveltimes in an acoustic medium.

The problem of determining the Riemannian metric from first arrivals is known in differential geometry as the boundary rigidity problem. The travel time information is encoded in the boundary distance function, which measures the distance, with respect to the Riemannian metric, between boundary points. The problem of determining the index of refraction from multiple arrival times is called in differential geometry as the lens rigidity problem. The information is encoded in the scattering relation which gives the exit point and direction of a geodesic if we know the incoming point and direction plus also the travel time.

The boundary rigidity problem consists in determining a compact Riemannian manifold with boundary up to an action of a diffeomorphism which is the identity at the boundary by knowing the geodesic distance function between boundary points (see [47, 49] and references therein). One needs an a-priori hypothesis to do so since it is easy to find counterexamples if the index of refraction is too large in certain regions. An a-priori condition that has been proposed is simplicity of the metric [28]. A manifold is simple if the boundary is strictly convex with respect to the Riemannian metric and there are no conjugate points along any geodesic. We remark that for simple manifolds knowing the scattering relation is the same as knowing the boundary distance function. It is only for non-simple manifolds that the scattering relation gives more information including multiple arrival times. See $[51,52]$ for recent works for understanding conjugate points (caustics) and lens rigidity problems.

In [21], Kurylev, Lassas and Uhlmann have established an uniqueness result for recovering a compact Riemannian manifold from broken scattering relations. The essential idea in [21] is the following: first, they impose some conditions on the broken scattering relation to verify whether a given family of geodesics intersect at one point; second, they show that the broken scattering relation determines the boundary distance representation of the Riemannian manifold, and the uniqueness follows from a certain isomorphism. However, the method of proof for the uniqueness result is not constructive. Another difficulty in practice is how to distinguish broken and non-broken scattering relations in the measurements. Here we propose a numerical reconstruction algorithm which is able to utilize both broken and non-broken scattering relations accordingly. First, we formulate the following simplified problem: how to reconstruct index of refraction from reflection traveltimes based on given locations of reflectors, such as an embedded mirror inside the medium? Tacitly, we consider an incident ray path and its corresponding reflected ray path as a broken geodesic [21] with a reflection condition at the broken point. Next we consider the more challenging problem of generic reflection traveltime tomography in which an unknown reflector is buried in an unknown medium.

Numerically, since traveltime tomography has a long history of development, let us put our work under appropriate perspectives. In terms of source-receiver setup, traveltime tomography can be classified into transmission traveltime tomography, reflection traveltime tomography, or reflection 
plus transmission traveltime tomography $[60,11,59,10,3,1,18,29,41,42,22]$; see more references in the above citations. In terms of whether multipathing is allowed or not, traveltime tomography may be classified into first-arrival based traveltime tomography $[3,4,59,1]$ or multi-arrival based traveltime tomography $[53,13,2,5,6,23]$. In terms of how the forward modeling is carried out in the implementation process, traveltime tomography may be classified into Lagrangian ray-tracing ODE based traveltime tomography $[60,59,10,3,4,1,18,29]$ or Eulerian PDE-based traveltime tomography $[41,42,22,23]$. In terms of media under consideration, traveltime tomography may be classified into isotropic or anisotropic traveltime tomography.

In general, ray-tracing based first-arrival traveltime tomography is not robust because the ray path followed by ray tracing might not yield the least traveltime between a given source-receiver pair when there are multiple possible rays to connect the source-receiver pair in the presence of triplication and caustics. To develop a robust first-arrival based traveltime tomography, one has to first develop robust forward modeling methods to generate reliable first-arrival traveltimes between a given source-receiver pair. Based on the contemporary viscosity-solution theory for HamiltonJacobi equations $[24,8,9,7]$, a lot of efforts were devoted to developing fast and efficient eikonal solvers to compute first-arrivals for both isotropic media $[58,32,57,38,40,33,34,61,20,36$, 14, 26] and for anisotropic media [12, 35, 56, 20, 37, 19]. Among these finite-difference eikonal solvers, the first-order fast marching and the first-order fast sweeping methods have proved to be unconditionally stable. Furthermore, related to the work in [41, 42], fast-sweeping-based eikonal solvers have been successfully used in isotropic transmission traveltime tomography in [22], and the resulting method is robust; this work has been further developed in $[55,54]$ for three-dimensional practical data.

As the first-arrival based traveltime tomography has limited resolution when the to-be-imaged structure is very complicated, it is desirable to develop a systematic formulation to utilize all the arrivals between a source-receiver pair. One question immediately comes up: how to parametrize all the arrivals between a source and receiver pair so that the information can be encoded into a rigorous mathematical formulation? To do that, one has to use a phase-space formulation so that multiple arrivals resulting from multipathing can be parametrized naturally by initial ray directions. In other words, one has to make use of scattering relation to develop a rigorous mathematical framework. Such efforts have been made in [53, 13, 2, 5, 23, 6]. In [13], Delprat-Jannaud and Lailly have developed a phase-space approach to parametrize multi-arrival traveltimes by using the receiver location and the ray parameter at the receiver, and their implementation is based on Lagrangian ray-tracing ODE methods for forward modeling. In [23], Leung and Qian have developed a Liouville-equation-based PDE approach for carrying out traveltime tomography which is able to utilize all arrivals. In [2] Billette and Lambare have developed a phase-space approach which makes full of the scattering relation; however, the proposed model in [2] is not well justified yet as it includes not only the velocity (the metric) but also other parameters.

In $[5,6]$, Chung, Qian, Uhlmann and Zhao have developed a systematic phase-space approach for traveltime tomography for acoustic and elastic media by using the Stefanov-Uhlmann identity (10) formulated in [44]. The advantages of the approach in [5,6] are multifold. As a first advantage multipathing can be taken into account systematically, as evidenced in [13, 23] and in numerical examples shown later. As demonstrated in [16, 25], multipathing is essential for high resolution seismic imaging. As a second advantage, our phase space formulation has the potential to recover generic (anisotropic) Riemannian metrics. These advantages distinguish our new method from 
other traditional methods in inverse kinematic problems [3, 39, 41, 42, 4, 59, 22] in that those traditional methods only recover isotropic metrics by using first-arrivals. Moreover, our numerical algorithm is based on a hybrid approach. A Lagrangian formulation (ray tracing) is used in phase space for the linearized Stefanov-Uhlmann identity (11). This allows us to deal with multipathing naturally. On the other hand, an Eulerian formulation is used for the index of refraction of the medium. As a consequence our computational domain is in physical space rather than in phase space, which reduces the degree of freedom and hence the computation cost.

Because the Stefanov-Uhlmann identity is posed in phase space, we have to find a way to pick the data that are in phase space, and such data are not measurable directly. To do that we recall that in kinematic inverse problems, the data used frequently is traveltime data, which means that traveltimes can be parametrized by source locations and ray parameters; in turn ray parameters can be derived from the eikonal equation and the traveltime data as illustrated in $[27,17,53,23]$. Therefore without any hesitation we use the identity as our foundation to carry out the inversion process.

The Stefanov-Uhlmann identity is also related to the so-called Liouville equation, but the current formulation is different from the one used in [23]. In that work [23] Leung and Qian formulated the inverse problem for isotropic metrics in an Eulerian framework and used an adjoint state method to minimize a mismatching functional. The current new formulation is based on a novel identity to cross-correlate the information from two metrics so that the two metrics can pass information to each other at every stage.

In this work we improve the phase space method developed in [5] by incorporating an adaptive strategy into the formulation. Although the Stefanov-Uhlmann identity (10), which links two metrics and their corresponding scattering relations together, is valid in quite general setting, the identity is truly nonlinear in terms of the two metrics. It is essential that the two metrics are close to each other in both mathematical analysis and numerical computation (through linearization). For the phase space method proposed in [5], all geodesics are used simultaneously based on the linearized Stefanov-Uhlmann identity (11) at each step. First, this creates a large linear system that involves the unknowns in the computational domain. Second, when an initial guess of the metric is poor, use of predicted geodesics that are far from the true ones may lead to too many iterations or even make the iterative procedure diverge. The key idea of the adaptive approach is to first utilize those geodesics that match the measurements well under the current metric. For example, geodesics that are short enough can always match the data well. We then use the hybrid phase space method restricted to these geodesics to improve/recover the metrics in the neighborhood of these geodesics in the physical space. As a result of the improved approximation of the metric in a certain part of the domain, more geodesics will match with the measurements better and will be used at the next step. If we continue with the process, more geodesics will be used so that one can recover the metrics in a larger region of the domain. The adaptive approach improves stability by using only those more accurate geodesics at each step. It also improves efficiency by gradually involving more and more unknowns in a stable fashion. A physical analog could be in the spirit of layer stripping. Initially short geodesics which are usually close to the boundary are used to provide a good estimate of the metric in a boundary layer. Then longer geodesics are used and the boundary layer of good estimate expands further into the interior. Of course the crucial point is that we do not have to specify the layers physically which is impossible without knowing the underlying metric. Instead we use data matching to automatically pick geodesics sequentially and our hybrid 
phase space method can recover the underlying metric in the neighborhood of the picked geodesics in physical space. Also geodesics that match data well may not be short ones. We then apply this adaptive phase space method to reflection tomography where broken geodesics/rays have to be taken into account. In particular, a jump condition of the geodesic flow in the phase space has to be enforced at the broken point and the Stefanov-Uhlmann identity has to be modified accordingly. More importantly our adaptive strategy can effectively distinguish and utilize measurements from non-broken and broken geodesics accordingly.

The paper is organized as follows: we introduce the formulation for reflection traveltime tomography and broken geodesics in Section 2. Then we present the numerical algorithm and the adaptive approach in Section 3. Numerical examples are presented in Section 4.

\section{Mathematical formulation for reflection traveltime tomography}

Let $\Omega$ be a bounded domain in $\mathbb{R}^{n}$ and let $\left(g_{i j}\right)$ be a Riemannian metric defined on it. Following $[5,6]$, we define the Hamiltonian $H_{g}$ by

$$
H_{g}(x, \xi)=\frac{1}{2}\left(\sum_{i, j=1}^{n} g^{i j}(x) \xi_{i} \xi_{j}-1\right)
$$

for each $x \in \Omega$ and $\xi \in \mathbb{R}^{n}$. In the above definition $\left(g^{i j}\right)=\left(g_{i j}\right)^{-1}$ is the inverse of the matrix $\left(g_{i j}\right)$. Let $X^{(0)}=\left(x^{(0)}, \xi^{(0)}\right)$ be a given initial condition from the following inflow set

$$
\mathcal{S}^{-}=\left\{(x, \xi) \mid x \in \partial \Omega, H(x, \xi)=1, \sum_{i, j=1}^{n} g^{i j}(x) \xi_{i} \nu_{j}(x)<0\right\}
$$

where $\nu(x)$ is the unit outward normal vector of $\partial \Omega$ at the point $x$ and $\nu_{j}(x)$ denotes the $j$-th component of this vector. We define $X_{g}\left(s, X^{(0)}\right)=(x(s), \xi(s))$ by the solution of the following system

$$
\frac{d x}{d s}=\frac{\partial H_{g}}{\partial \xi}, \quad \frac{d \xi}{d s}=-\frac{\partial H_{g}}{\partial x}
$$

with the initial condition

$$
(x(0), \xi(0))=X^{(0)} .
$$

We suppress the dependence of $(x(s), \xi(s))$ on $X^{(0)}$. The solution $X_{g}$ defines a geodesic/ray in the phase space, parametrically via $x(s)$, in the physical space $\Omega$ with co-tangent vector $\xi(s)$ at any point $x(s)$. The parameter $s$ denotes travel time.

In this paper, we consider the case when there are obstacles inside the domain $\Omega$. In this case, the ray will be reflected at the boundary of the obstacles. Mathematically, we need to impose jump condition for the above system (2). For simplicity, we will derive the jump condition for the case when there is only one obstacle lying strictly inside $\Omega$ and the ray intersects the obstacle at most once. Let $\Gamma$ be the interface where the ray will be reflected. Notice that there is a unique time $s^{*}>0$ such that the point $x\left(s^{*}\right)$ hits the interface at an incoming angle defined by $\xi_{\text {in }}:=\xi\left(s^{*}\right)$. The ray will be reflected at an outgoing angle defined by $\xi_{\text {out }}=R\left(\xi_{\text {in }} ; x\left(s^{*}\right)\right)$ which is specified by 
the normal vector of $\Gamma$ at the point $x\left(s^{*}\right)$ according to the law of reflection in geometrical optics. We remark that the function $R$ depends on the contact point $x\left(s^{*}\right)$.

Thus, the vector $X_{g}\left(s, X^{(0)}\right)$ is defined by the following

$$
\begin{aligned}
& \frac{d x}{d s}=\frac{\partial H_{g}}{\partial \xi}, \quad \frac{d \xi}{d s}=-\frac{\partial H_{g}}{\partial x}, \quad 0<s<s^{*}, \\
& (x(0), \xi(0))=X^{(0)}
\end{aligned}
$$

and

$$
\begin{aligned}
& \frac{d x}{d s}=\frac{\partial H_{g}}{\partial \xi}, \quad \frac{d \xi}{d s}=-\frac{\partial H_{g}}{\partial x}, \quad s>s^{*}, \\
& \left(x\left(s^{*}\right), \xi\left(s^{*}\right)\right)=\left(x\left(s^{*}\right), \xi_{\text {out }}\right) .
\end{aligned}
$$

In the derivation below, we will need the Jacobian matrix

$$
J_{g}\left(s, X^{(0)}\right):=\frac{\partial X_{g}}{\partial X^{(0)}}\left(s, X^{(0)}\right)=\left(\begin{array}{ll}
\frac{\partial x}{\partial x^{(0)}} & \frac{\partial x}{\partial \xi^{(0)}} \\
\frac{\partial \xi}{\partial x^{(0)}} & \frac{\partial \xi}{\partial \xi^{(0)}}
\end{array}\right)
$$

which is the derivative of $X_{g}$ with respect to the initial condition $X^{(0)}$. Let

$$
M=\left(\begin{array}{cc}
H_{\xi, x} & H_{\xi, \xi} \\
-H_{x, x} & -H_{x, \xi}
\end{array}\right)
$$

Then we have

$$
\frac{d J}{d s}=M J, \quad J(0)=I, \quad \text { for } 0<s<s^{*},
$$

and

$$
\frac{d J}{d s}=M J, \quad J\left(s^{*}\right)=B, \quad \text { for } s>s^{*},
$$

where

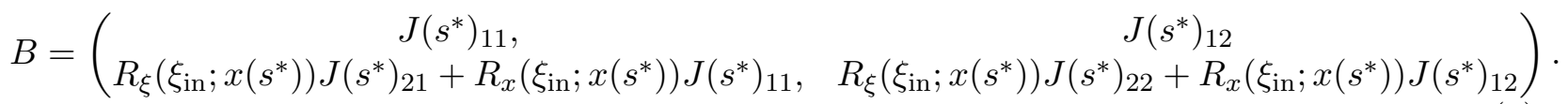

Next we will derive the broken Stefanov-Uhlmann identity. Similar to [44, 5], we define

$$
F(s)=X_{g_{2}}\left(t-s, X_{g_{1}}\left(s, X^{(0)}\right)\right)
$$

where $t=t_{g_{1}}$. Then we have

$$
\int_{0}^{t} F^{\prime}(s) d s=X_{g_{1}}\left(t, X^{(0)}\right)-X_{g_{2}}\left(t, X^{(0)}\right) .
$$

The time integral on the left hand side is

$$
\int_{0}^{t} F^{\prime}(s) d s=\int_{0}^{t} \frac{\partial X_{g_{2}}}{\partial X^{(0)}}\left(t-s, X_{g_{1}}\left(s, X^{(0)}\right)\right) \times\left(V_{g_{1}}-V_{g_{2}}\right)\left(X_{g_{1}}\left(s, X^{(0)}\right)\right) d s .
$$


Hence we have the Stefanov-Uhlmann identity

$$
X_{g_{1}}\left(t, X^{(0)}\right)-X_{g_{2}}\left(t, X^{(0)}\right)=\int_{0}^{t} J_{g_{2}}\left(t-s, X_{g_{1}}\left(s, X^{(0)}\right)\right) \times\left(V_{g_{1}}-V_{g_{2}}\right)\left(X_{g_{1}}\left(s, X^{(0)}\right)\right) d s .
$$

Linearizing the right hand side at $g_{2}$, we have

$$
X_{g_{1}}\left(t, X^{(0)}\right)-X_{g_{2}}\left(t, X^{(0)}\right) \approx \int_{0}^{t} J_{g_{2}}\left(t-s, X_{g_{2}}\left(s, X^{(0)}\right)\right) \times \partial_{g_{2}} V_{g_{2}}\left(g_{1}-g_{2}\right)\left(X_{g_{2}}\left(s, X^{(0)}\right)\right) d s .
$$

In the case of an isotropic medium

$$
g_{i j}=\frac{1}{c^{2}} \delta_{i j}, \quad \partial_{g} V_{g}(\lambda)=\left(2 c \lambda \xi,-(\lambda \nabla c+c \nabla \lambda)|\xi|^{2}\right) .
$$

Moreover, we have the following group property

$$
J_{g_{2}}\left(t-s, X_{g_{2}}\left(s, X^{(0)}\right)\right)=J_{g_{2}}\left(t, X^{(0)}\right) J_{g_{2}}\left(s, X^{(0)}\right)^{-1} .
$$

\section{Adaptive phase space method}

\subsection{The phase space method}

We first introduce the general setup of the phase space method for traveltime tomography proposed in [5]. The numerical method is an iterative algorithm based on the linearized Stefanov-Uhlmann identity (11) using a hybrid approach. The metric $g$ is defined on an underlying Eulerian grid in the physical domain. The integral equation (11) is discretized along a ray for each $X^{(0)}$ in phase space. The Jacobian matrix along the ray is computed according to (7) (and (8), (9) for broken rays). On the ray, values of $g$ are computed by interpolation from the neighboring grid point values. Hence each integral equation along a particular ray yields a linear equation for grid values of $g$ in the neighborhood of the ray in the physical domain (see Figure 3.1). Here is the iterative algorithm for finding $g$.

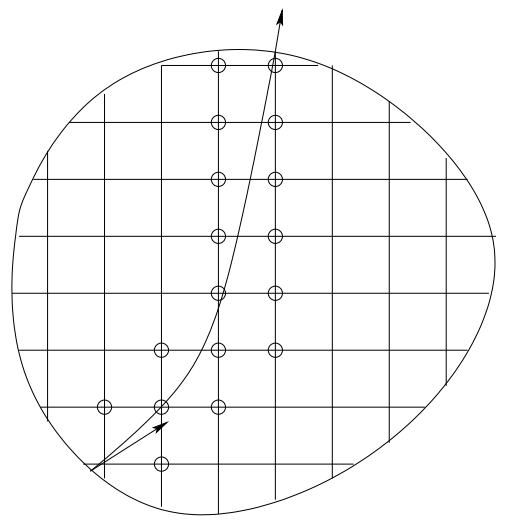

Let $X_{i}^{(0)}, i=1,2, \cdots, m$, be the initial locations and directions of those $m$ measurements (scattering relations) $X_{g}\left(t_{i}, X_{i}^{(0)}\right)$ where $t_{i}$ is the exit time corresponding to the $i$-th geodesic starting at $X_{i}^{(0)}$. Starting with an initial guess of the metric $g^{0}$, we construct a squence $g^{n}$ as follows. 
Define the mismatch vector

$$
d_{i}^{n}=X_{g}\left(t_{i}, X_{i}^{(0)}\right)-X_{g^{n}}\left(t_{i}, X_{i}^{(0)}\right)
$$

and a linear operator based on (11) along $i$-th geodesic

$$
K_{i}^{n} g=\int_{0}^{t_{i}} J_{g^{n}}\left(t_{i}-s, X_{g^{n}}\left(s, X_{i}^{(0)}\right)\right) \times \partial_{g^{n}} V_{g^{n}}(g)\left(X_{g^{n}}\left(s, X_{i}^{(0)}\right)\right) d s .
$$

Notice that both $d_{i}^{n}$ and $K_{i}^{n}$ depend on $X_{i}^{(0)}$. Then, for each $n \geq 0$, we find $\tilde{g}$ that minimizes

$$
F(g)=\frac{1}{2} \sum_{i=1}^{m}\left\|K_{i}^{n} g-d_{i}^{n}\right\|^{2}+\frac{\beta}{2}\|\nabla g\|_{L^{2}(\Omega)}^{2},
$$

where the last term is a regularization term since the inverse problem is ill-posedness and the resulting linear system may not have a unique solution. The choice of $\beta$ may depend on the noise level and scale of the problem. We then define

$$
g^{n+1}=g^{n}+\tilde{g}
$$

\subsection{An adaptive strategy}

In the original phase space method all rays from the measurements are used at the same time for the reconstruction; see equations (12) and (13). It results in a large linear system. Moreover, use of geodesics that are far from the true ones may make the linearization based iterative algorithm converge slowly with more iterations or even make the algorithm diverge. So we propose the following adaptive strategy. At each step we pick up those geodesics in the current guessed metric that produce small mismatch with the measurements, i.e., the scattering relations. Then we apply the phase space method only to these geodesics which will provide a good approximation of the true metric in the neighborhood of those picked geodesics. Then the improvement of the guessed metric will result in more geodesics that have small mismatch with measurements; in turn, this allows us to recover the metric in a larger region in the next step.

Here is the adaptive algorithm we used in our numerical implementation. For each $n$, we will use $D_{n}$ to represent a subset of $\{1,2, \cdots, m\}$. The set $D_{n}$ contains all indices $i$ such that the mismatch data $d_{i}^{n}$ are small enough. More precisely, for a given tolerance $\varepsilon>0$, we define

$$
D_{n}=\left\{i \mid \frac{\left\|d_{i}^{n}\right\|}{\left\|X_{g}\left(t_{i}, X_{i}^{(0)}\right)-X_{i}^{(0)}\right\|}<\varepsilon\right\},
$$

where the mismatch is normalized by the difference between the starting location and ending location for each geodesic in the phase space, which can be obtained directly from the measurements. Then, for each $n \geq 0$, we find $\tilde{g}$ that minimizes

$$
F(g)=\frac{1}{2} \sum_{i \in D_{n}}\left\|K_{i}^{n} g-d_{i}^{n}\right\|^{2}+\frac{\beta}{2}\|\nabla g\|^{2} .
$$


We then define

$$
g^{n+1}=g^{n}+\tilde{g} .
$$

In practice, we will choose $\varepsilon$ to be proportional to the sum of normalized mismatch $\sigma_{n}$ defined by

$$
\sigma_{n}=\sum_{i=1}^{m} \frac{\left\|d_{i}^{n}\right\|}{\left\|X_{g}\left(t_{i}, X_{i}^{(0)}\right)-X_{i}^{(0)}\right\|} .
$$

This adaptive strategy has some flavor of layer stripping method in the sense that shorter geodesics that are closer to the boundary usually give smaller mismatch and are used at an earlier stage to recover/improve the metric near boundary. However, the crucial difference is that our method does not need to define layers explicitly in physical space and is done automatically based on data. This adaptive approach improves robustness, efficiency and accuracy in comparison to using all geodesics simultaneously.

Moreover, the adaptive strategy can distinguish and use broken geodesics and non-broken geodesics in the measurements, which is important for reflection travel time tomography. On one hand, if the obstacle or the reflection interface is inside the domain, using broken geodesics only may be unstable since there may be no short broken geodesics that is a small perturbation of the true one. On the other hand, using non-broken geodesics only can at most recover the metric outside the convex (with respect to the metric) hull of the obstacle. Nevertheless, our adaptive strategy can distinguish broken and non-broken geodesics when both are present in the measurements. Because if we predict a broken or non-broken geodesic erroneously, it will produce a large error $(\mathrm{O}(1))$ in the scattering relation due to the jump condition (9) at the broken point. Hence incorrectly predicted geodesics will not be used in the reconstruction. In practice, the adaptive strategy will likely pick up those non-broken and short geodesics that provide small mismatch and recover the metric in a region close to the boundary first. Then more and more geodesics, including broken ones, will be picked up so that larger and larger region, including the concave region, will be covered. These observations are verified by numerical results in the next section. Actually we show that we can use geodesics that are broken for multiple times numerically. In the most challenging situation where neither the obstacle nor the underlying metric is known, our adaptive method can pick up most non-broken geodesics during the reconstruction. As a result (see Example 4.5 below) we recover: (1) the convex hull of the unknown obstacle given by the envelope of used non-broken geodesics, and (2) the metric outside the convex hull. An interesting problem for future study is how to improve the reconstruction using broken geodesics based on the result from non-broken ones. The main challenge is how to represent the geometry of the envelope of the used non-broken geodesics to predict the broken points and jump conditions for broken geodesics.

\section{$4 \quad$ Numerical experiments}

In this section, we use numerical experiments to show effectiveness and robustness of our adaptive approach in various setups.

\subsection{Example 1. An example with no broken geodesic}

The purpose of this example is to compare the reconstruction obtained by using all geodesics simultaneously (the original phase space method proposed in [5]) with that obtained by the adaptive 
strategy proposed in this work.

The exact solution is $c(x, y)=1+0.3 \sin (2 \pi x) \sin (2 \pi y)$. The initial guess is $c_{0}(x, y)=0.8$. The grid size is $20 \times 20$ and we use 200 directions at each grid point on only one side of the boundary: $\{y=0\}$. The regularization parameter is taken as $\beta=0.1$.

In the adaptive approach, we take $\varepsilon=0.25 \sigma_{n}$. The algorithm converges to a solution with a relative error $1.3 \times 10^{-2}$ at the 55 -th iteration. We plot numerical and exact solutions in the left plot and the center plot of Figure 1, respectively. We see that we obtain a good recovery of the unknown function $c(x, y)$.

Without the adaptive strategy the algorithm converges at the 67 -th iteration with a relative error $4.4 \times 10^{-2}$. The numerical solution in this case is shown in the right plot of Figure 1 .
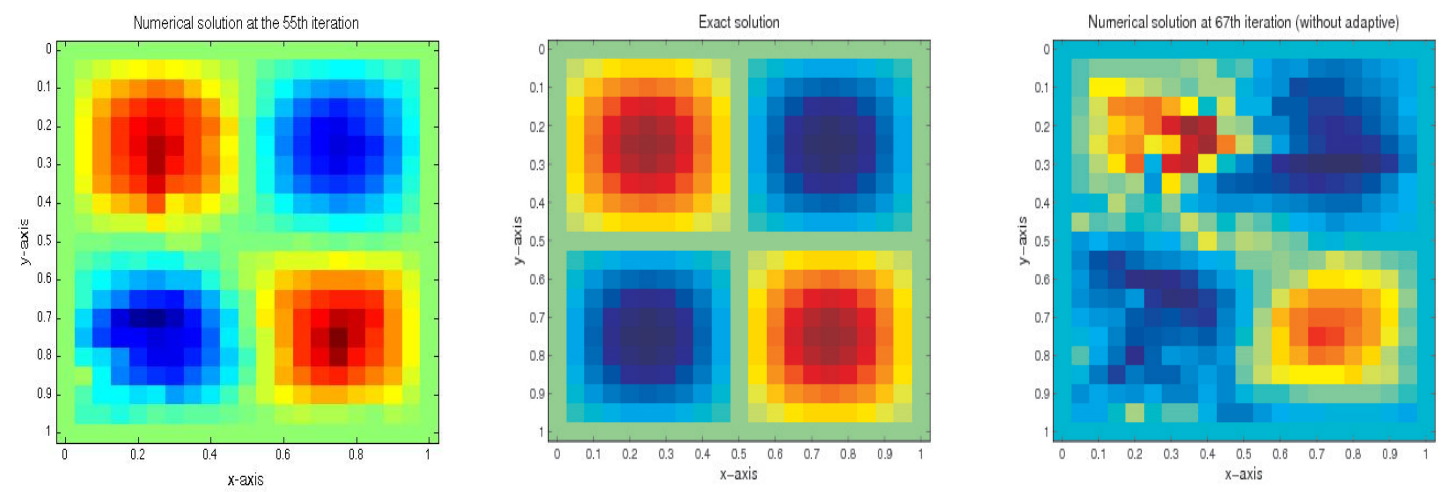

Figure 1: Left: Numerical solution (using adaptive) at the 55-th iteration. Middle: Exact solution. Right: Numerical solution (without adaptive) at the 67-th iteration.

\subsection{Example 2. A known circular obstacle enclosed by a square domain}

We consider a known circular obstacle with boundary $\Gamma$ being a circle with center $(0.5,0.5)$ and radius 0.3 . In this example the geodesic either does not hit the inclusion (non-broken) or hits the inclusion (broken) once.

The exact solution is $c(x, y)=1+\frac{1}{5} \sin (2 \pi x) \sin (\pi y)$. The initial guess is $c_{0}(x, y)=0.8$. The grid size is $20 \times 20$ and we use 100 incoming directions at each grid point on the boundary. We also add $5 \%$ noise to the data. The regularization parameter is taken as $\beta=10^{-4}$. For the parameter of the adaptive strategy, we take $\varepsilon=0.5 \sigma_{n}$. The algorithm converges to a solution with a relative error $9.4 \times 10^{-4}$ at the 20-th iteration. We plot the numerical and exact solutions in Figure 2, respectively.

\subsection{Example 3. A circular domain}

We consider the case when the domain $\Omega$ is a circle which is centered at $(0.5,0.5)$ with radius 0.4.

The exact solution is $c(x, y)=1+0.3 \cos (r)$ where $r=\sqrt{(x-0.5)^{2}+(y-0.5)^{2}}$. The initial guess is $c_{0}(x, y)=0.6$. The grid size is $40 \times 40$. We choose 30 source points which are uniformly 

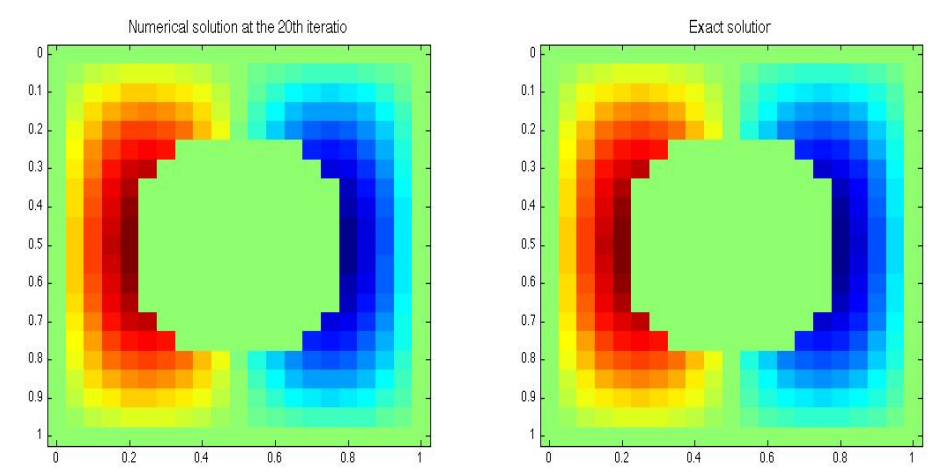

Figure 2: Left: Numerical solution at the 20-th iteration. The relative error is $0.094 \%$. Right: Exact solution.

distributed on the boundary of $\Omega$ and at each source point we use 101 incoming directions. The regularization parameter $\beta=10^{-3}$. For the parameter of the adaptive process, we take $\varepsilon=0.05 \sigma_{n}$.

The numerical results of the first six iterations are shown in Figure 3. The ray coverage for the first six iterations are shown in Figure 4. The number of rays used are 478,620,900, 1117, 1406 and 1625, respectively. We can see that the adaptive strategy picks up mostly short geodesics first. As the iteration goes on, more and more geodesics are picked up that cover larger and larger regions to update the metric.

In Figure 5, we have shown both the numerical solution at the 13-th iteration and the exact solution. The two match with each other very well with relative error $0.01 \%$.

\subsection{Example 4. A concave obstacle}

In this example, we consider the numerical reconstruction of an unknown medium which contains a known concave obstacle. In this case, some geodesics can have more than one reflections at the obstacle interface, which poses a challenge in the numerical reconstruction.

The exact solution is $c(x, y)=1+0.1 \sin (0.5 \pi x) \sin (0.5 \pi y)$. The initial guess is $c_{0}(x, y)=0.8$. The grid is $30 \times 30$ and we use 30 directions at each grid point on the boundary. The regularization parameter is taken as $\beta=1$. For the parameter in the adaptive approach, we take $\varepsilon=\min \left(0.5 \sigma_{n}, 1\right)$.

The inclusion is formed by a concave kite shape object with the parametric representation

$$
x(t)=\cos (t)+0.65 \cos (2 t)-0.65, \quad y(t)=1.5 \cos (t), \quad 0 \leq t \leq 2 \pi .
$$

In this case, there are geodesics that have 4 reflections at the interface in our simulation.

Our adaptive numerical algorithm gives a numerical approximation at the 117 iteration with a relative error of $2.8 \%$. Without the adaptive strategy, the phase space method does not even converge for this example. The numerical results are shown in Figure 6. In Figure 7, we show rays that are not used (left) and broken rays that are used (right) in the calculation. We see that most non-broken rays are used in the final reconstruction and most of the unused rays have reflections in the concave region. As discussed before, the difficulty at concave region is due to the fact that neither non-broken geodesics nor short broken geodesics can reach the concave region. On the 
Numerical solution at the 2nd iteration
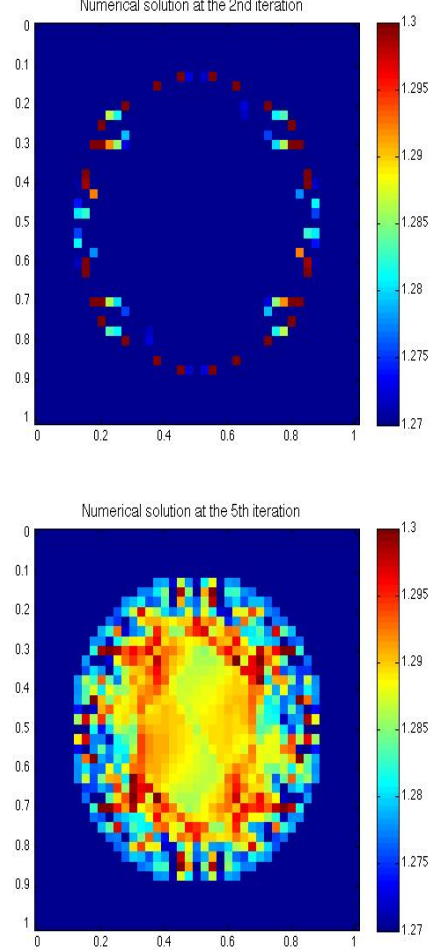

Numerical solution at the 3rd teration
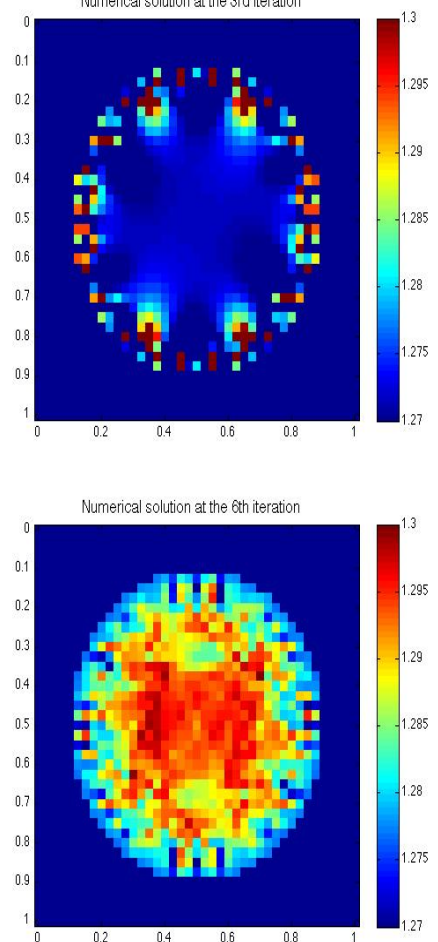

Numerical solution at the the iteration
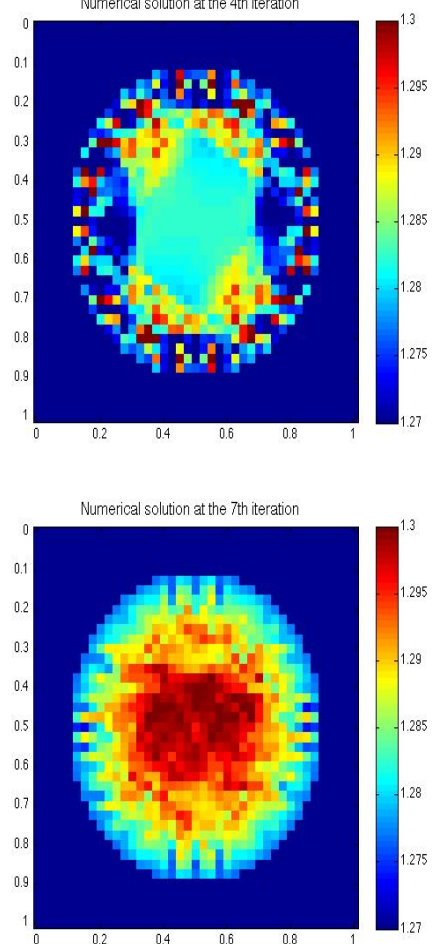

Figure 3: Numerical solutions from the second to the seventh iterations.

other hand broken geodesics have to be used to reach concave region. In the adaptive strategy, after non-broken rays are used to provide a good estimate of the metric outside the convex hull of the concave region, some broken rays are then used to provide estimate of the metric inside the concave region. Although many broken rays are not used, we still manage to get a pretty good reconstruction in the concave region. By avoiding using those erroneous rays in our adaptive approach, we gain stability. Of course the tradeoff between accuracy and stability is always a tricky issue. In general, concave region in reflection tomography poses a great challenge due to multiple reflection or scattering; the more concave the region is the more difficult it is to reconstruct.

\subsection{Example 5. Unknown obstacles}

In this section, we present a few examples in the most difficult setting in reflection traveltime tomography where both the location of reflection and the underlying medium are unknown. We demonstrate that our adaptive method is able to recover the convex (with respect to the underlying metric) hull of the unknown concave obstacles and the metric outside the convex hull. The key idea is that our adaptive method can distinguish non-broken rays from broken ones in the measurements and use non-broken ones for the reconstruction first. Since we do not know whether there is reflection or not and we do not know the location of the reflection if there is, we can not use those broken geodesics. Hence we assume that there is no reflection first. So those rays that are broken will not be used in the reconstruction because the erroneous assumption misses the jump condition 

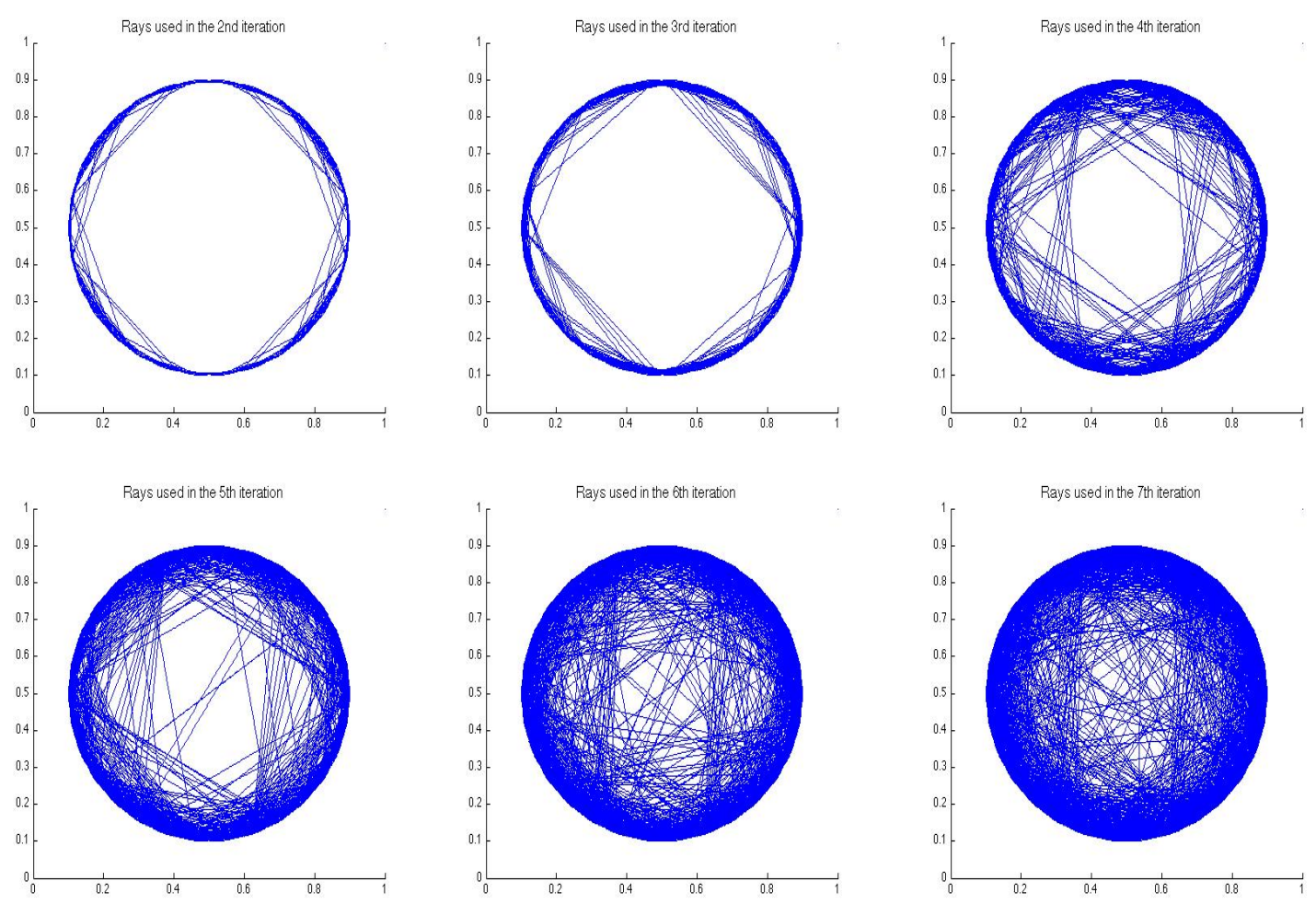

Figure 4: Rays used from the second to the seventh iterations.

at the broken point which will produce a large mismatch with the measurement. Once most nonbroken rays are used we can (1) plot those rays to find the convex hull of the obstacle (if there is one), and (2) reconstruct the metric outside the convex hull.

In the first two examples (Figures 8-9), we show recovery of obstacles with an unknown constant velocity field $c(x)=1$. Then we will present an example (see Figure 10) with non-constant velocity field with a convex unknown obstacle. In the final example (see Figures 11-12), we will consider the recovery of both the non-convex unknown obstacle and the unknown non-constant velocity field.

In the first test, we use an unknown obstacle of the concave kite shape as in Section 4.4. In Figure 8, we show a figure where we draw only those rays that are used in the calculation. We see that the rays give the convex hull of the unknown concave obstacle.

In Figure 9, we show another test where there are two unknown concave obstacles. The two obstacles are shown in the left plot of Figure 9. In the middle plot of Figure 9, we plot the rays that are used in the reconstruction. Again we obtain the convex hull for each of the two obstacles because they are well separated. Moreover, the relative error of the recovered velocity field is only $0.3 \%$.

In Figure 10, we show the reconstruction with a convex unknown elliptical object defined by the equation $\frac{(x-0.5)^{2}}{0.04}+\frac{(y-0.5)^{2}}{0.09}=1$. The unknown velocity field is $c(x)=1-0.3 e^{-2 r^{2}}$ with $r=\sqrt{(x-0.5)^{2}+(y-0.5)^{2}}$. In the left plot of Figure 10, we present the rays that are used in the 

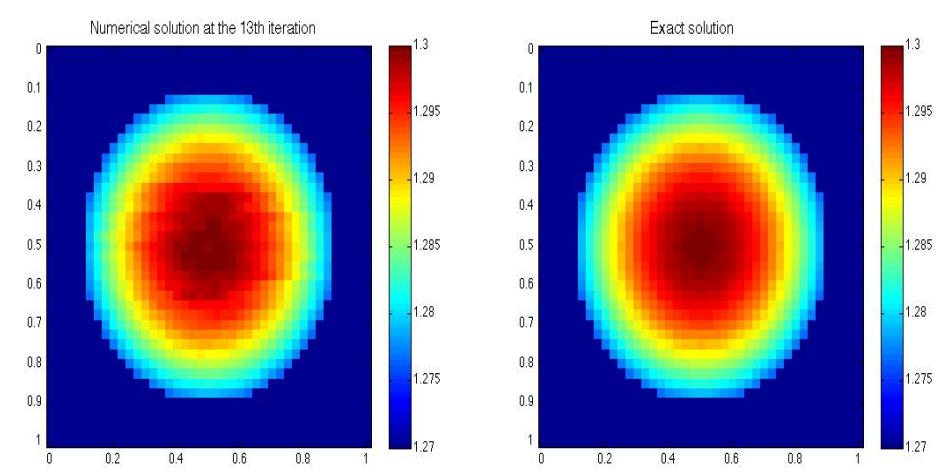

Figure 5: Left: Numerical solution at the 13-th iteration. Right: Exact solution.
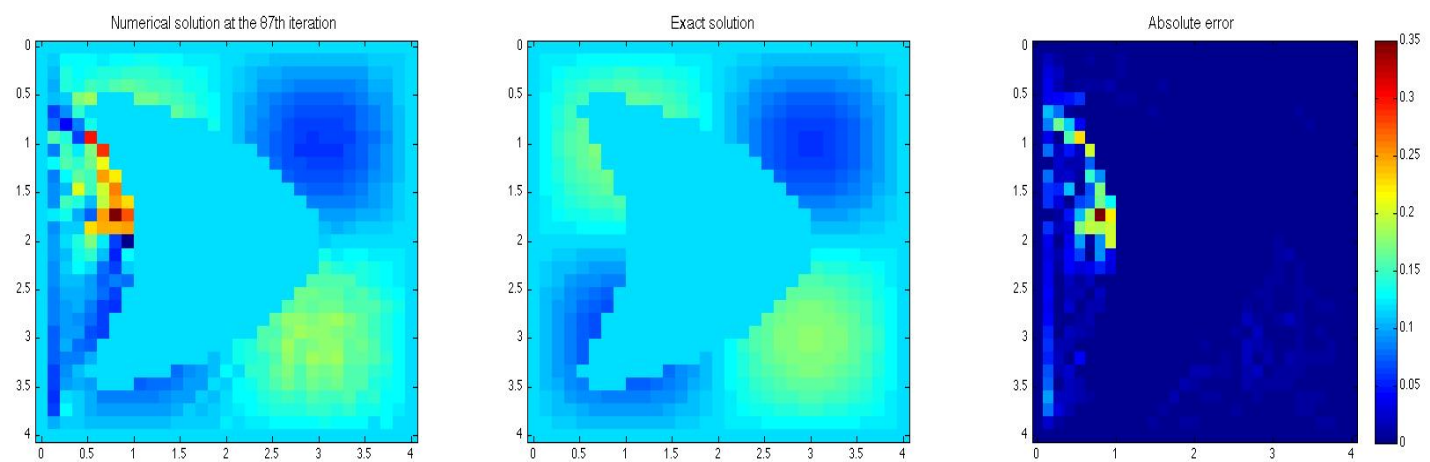

Figure 6: Left: Numerical solution at the 117-th iteration. The relative error is $2.8 \%$. Middle: Exact solution. Right: Absolute error.

calculation. One sees that the coverage of the rays gives an excellent reconstruction of the unknown obstacle. In the middle and right plots of Figure 10, we present both the numerical solution and the exact solution for the velocity field; the corresponding relative error is only $0.3 \%$.

In Figure 11, we show the reconstruction with a very concave unknown object of a flower shape $r=1+0.6 \cos (3 \theta)$ with $r=\sqrt{(x-2)^{2}+(y-2)^{2}}$. The unknown velocity field is $c(x)=1+0.2 \sin (r)$ with $r=\sqrt{(x-2)^{2}+(y-2)^{2}}$. The unknown obstacle is shown in the left plot of Figure 11. In this example, we also take the domain $\Omega$ as a circle. In the middle plot of Figure 11, we have shown the rays that are used in the calculation. Again we obtain the envelope of the convex hull of the obstacle. Absolute error of the numerical solution is also shown in the right plot of Figure 11. We see that we get a good recovery outside the convex hull of the unknown obstacle, which is the region covered by the rays. In Figure 12, we present both the numerical solution and the exact solution for the velocity field. The corresponding relative error is $2.67 \%$. 

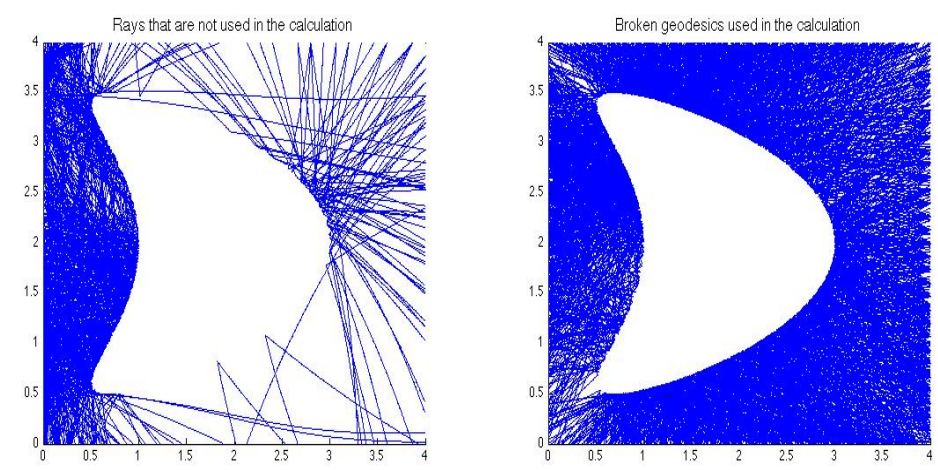

Figure 7: Left: Rays not used in the calculation. Right: Broken geodesics used in the calculation.

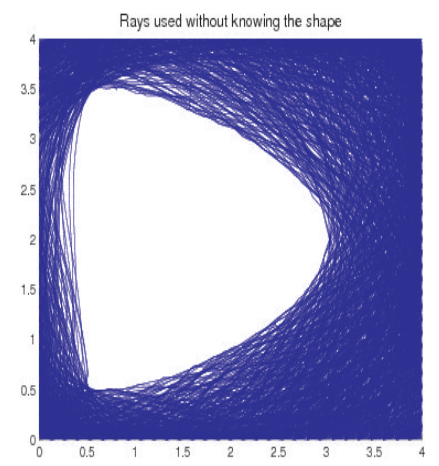

Figure 8: Ray coverage of the unknown obstacle.

\subsection{Example 6. The Marmousi model}

In this section, we test our method on a well-known bench mark problem: the Marmousi model. The exact velocity field is defined on a $122 \times 122$ grid and is shown in the left plot of Figure 13 . In our numerical reconstruction we use a $41 \times 41$ grid. We use 50 directions at each grid point along the boundary. The regularization parameter $\beta=100$. We will compare our numerical solution to the exact solution projected on a $41 \times 41$ coarse grid, see the middle plot of Figure 13 . We take the initial guess as $c_{0}=1500$. We use $\varepsilon=\min \left(0.1 \sigma_{n}, 1\right)$. The algorithm converges at the 16-th iteration with a relative error of $2.24 \%$. In the right plot of Figure 13, we have shown the numerical approximation. Comparing to the result we obtained using the original phase space method in [5] for the same problem, the adaptive method is much better

Furthermore, we test the robustness of our numerical algorithm by adding some noise in the data, with the same numerical setting as above except that we take $\beta=10000$. In Figure 14, we present the numerical solutions when the noise levels are $0.1 \%$ and $1 \%$, with relative errors $4.16 \%$ and $5.53 \%$ respectively. We see that our method behaves quite robustly. 

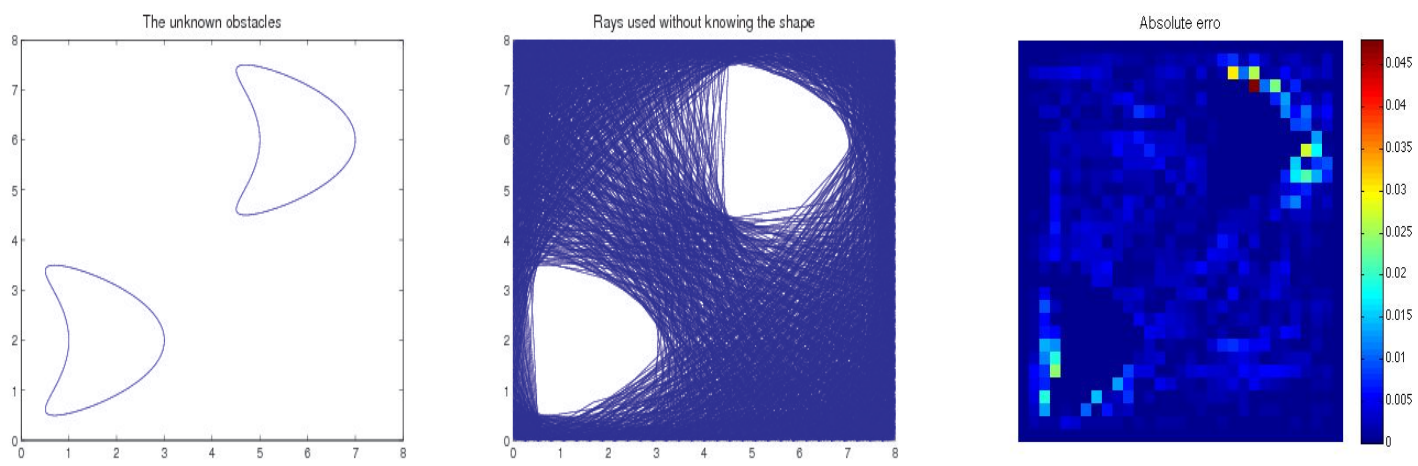

Figure 9: Left: The two unknown obstacles. Middle: Ray coverage of the unknown obstacle. Right: Absolute error.
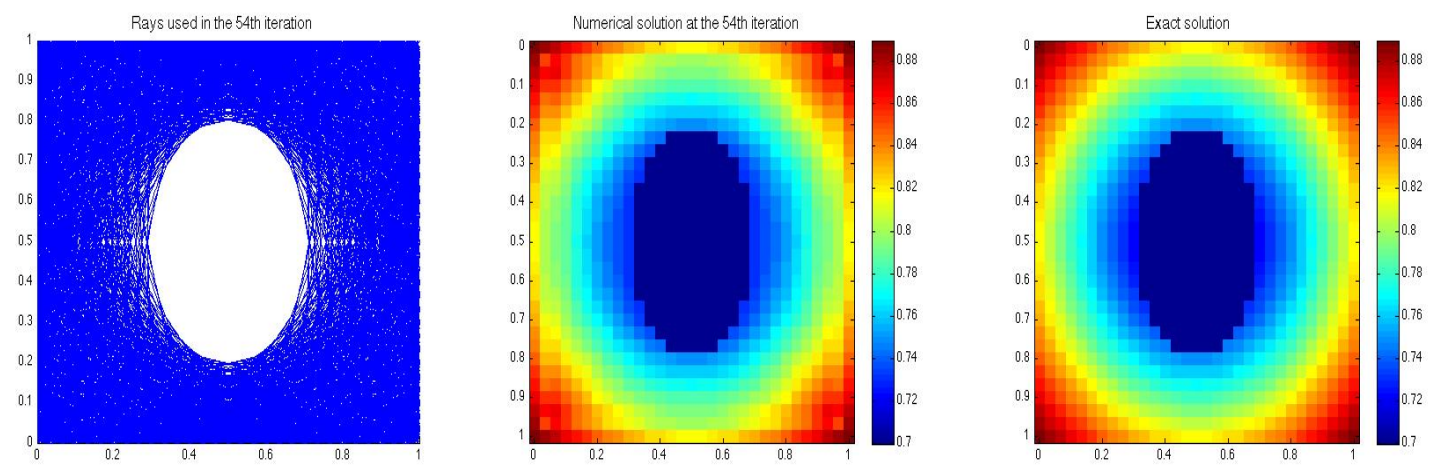

Figure 10: Left: Ray coverage of the unknown obstacle. Middle: Numerical solution at the 54-th iteration. Relative error is $0.3 \%$. Right: Exact solution.

\section{Conclusions}

In this work we presented an adaptive phase space method for traveltime tomography that can deal with multiple arrival time using the scattering relations. Compared to the original method proposed in [5], the adaptive strategy uses more accurate geodesics first and improves the reconstruction gradually in a more stable and efficient way. The proposed adaptive method can also distinguish and utilize broken and non-broken geodesics accordingly for the case of reflection traveltime tomography.

\section{Acknowledgement}

Chung is supported by RGC General Research Fund (Project Number 400609). Qian is supported by NSF 0810104. Uhlmann is partly supported by NSF a Chancellor Professorship at UC Berkeley and a Senior Clay Award. Zhao is supported by NSF Grant DMS0811254. 

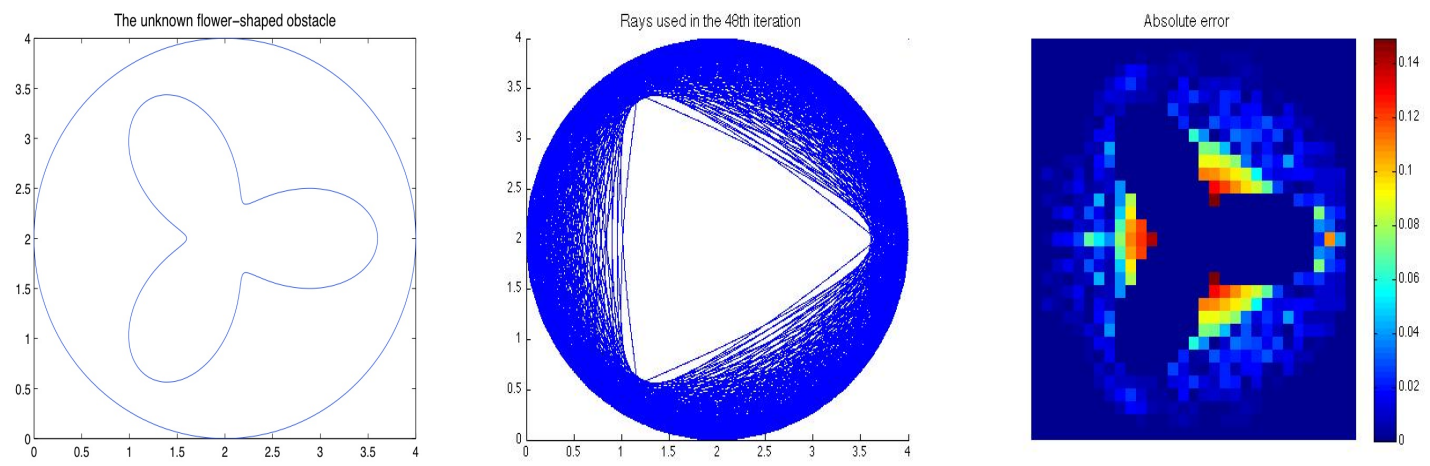

Figure 11: Left: The unknown obstacle. Middle: Ray coverage of the unknown obstacle. Right: Absolute error.
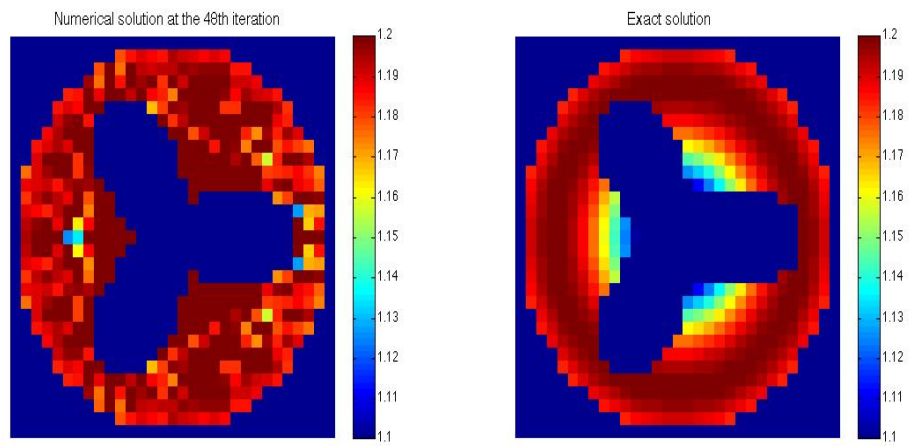

Figure 12: Left: Numerical solution at the 48-th iteration. Relative error is 2.67\%. Right: Exact solution.

\section{References}

[1] J. Berryman. Stable iterative reconstruction algorithm for nonlinear traveltime tomography. Inverse Problems, 6:21-42, 1990.

[2] F. Billette and G. Lambaré. Velocity macro-model estimation from seismic reflection data by stereotomography. Geophysical Journal International, 135:671-690, November 1998.

[3] T. N. Bishop, K. P. Bube, R. T. Cutler, R. T. Langan, P. L. Love, J. R. Resnick, R. T. Shuey, D. A. Spindler, and H. W. Wyld. Tomographic determination of velocity and depth in laterally varying media. Geophysics, 50:903-923, 1985.

[4] K. P. Bube and R. T. Langan. Hybrid $l^{1}-l^{2}$ minimization with applications to tomography. Geophysics, 62:1183-1195, 1997.

[5] E. Chung, J. Qian, G. Uhlmann, and H.K. Zhao. A new phase space method for recovering index of refraction from travel times. Inverse Problems, 23:309-329, 2007. 

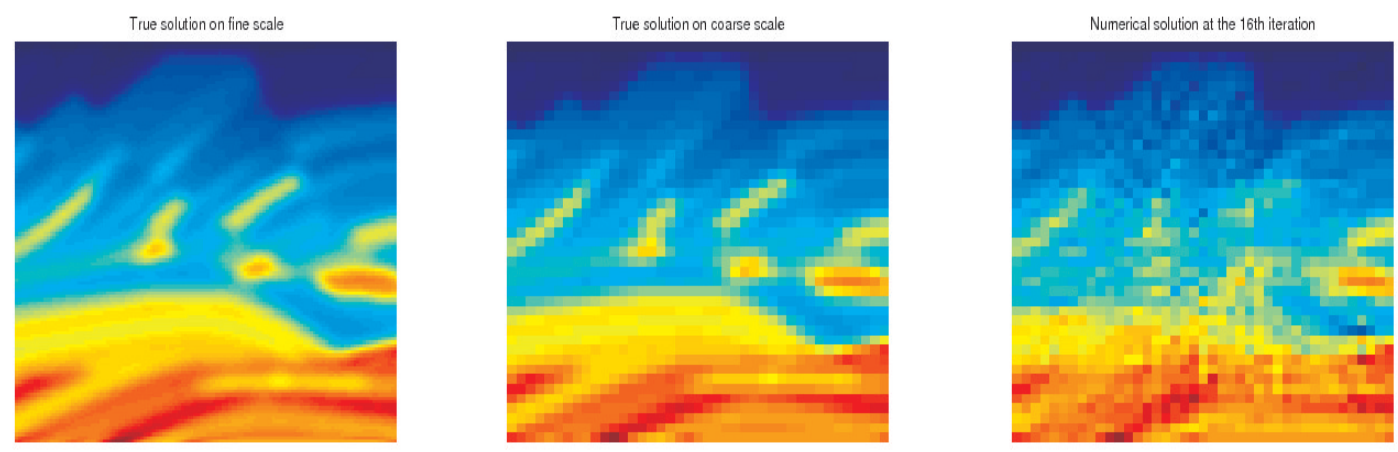

Figure 13: Marmousi model. Left: The exact solution on fine grid. Middle: The exact solution projected on a coarse grid. Right: The numerical solution at the 16-th iteration. The relative error is $2.24 \%$.
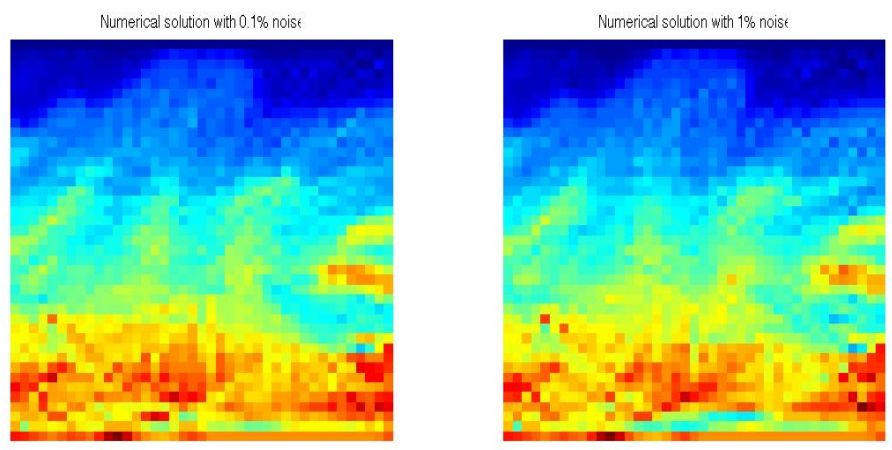

Figure 14: Marmousi model. Left: The numerical solution with $0.1 \%$ noise. The relative error is $4.16 \%$. Right: The numerical solution with $1 \%$ noise. The relative error is $5.53 \%$.

[6] E. Chung, J. Qian, G. Uhlmann, and H.K. Zhao. A phase-space formulation for elastic-wave traveltime tomography. J. Physics: Conference Series, 124:012018, 2008.

[7] M. G. Crandall, L. C. Evans, and P. L. Lions. Some properties of viscosity solutions of Hamilton-Jacobi equations. Trans. Amer. Math. Soc., 282:487-502, 1984.

[8] M. G. Crandall and P. L. Lions. Viscosity solutions of Hamilton-Jacobi equations. Trans. Amer. Math. Soc., 277:1-42, 1983.

[9] M. G. Crandall and P. L. Lions. Two approximations of solutions of Hamilton-Jacobi equations. Math. Comp., 43:1-19, 1984.

[10] F. A. Dahlen, S.-H. Hung, and G. Nolet. Frechet kernels for finite-frequency traveltimes- I. theory. Geophys. J. Int., 141:157-174, 2000. 
[11] A. J. Day, C. Peirce, and M. C. Sinha. Three-dimensional crustal structure and magma chamber geometry at the intermediate-spreading, back-arc Valu Fa Ridge, Lau Basin - results of a wide-angle seismic tomographic inversion. Geophys. J. Int., 146:31-52, 2001.

[12] J. Dellinger and W. W. Symes. Anisotropic finite-difference traveltimes using a HamiltonJacobi solver. In 67th Ann. Internat. Mtg., Soc. Expl. Geophys., Expanded Abstracts, pages 1786-1789. Soc. Expl. Geophys., Tulsa, OK., 1997.

[13] F. Delprat-Jannaud and P. Lailly. Reflection tomography: how to handle multiple arrivals? J. Geophys. Res., 100:703-715, 1995.

[14] S. Fomel, S. Luo, and H. K. Zhao. Fast sweeping method for the factored eikonal equation. J. Comput. Phys., 228:6440-6455, 2009.

[15] B. Frigyik, P. Stefanov, and G. Uhlmann. The x-ray transform for a generic family of curves and weights. J. Geom. Anal., 18:89-108, 2008.

[16] S. Gray. Efficient traveltime calculations for kirchhoff migration. Geophysics, 51:1685-1688, 1986.

[17] W. Harlan and R. Burridge. A tomographic velocity inversion for unstacked data. Stanford Exploration Project SEP37-01, 1983.

[18] J. M. Harris, R. C. Nolen-Hoeksema, R. T. Langan, M. Van Schaack, S. K. Lazaratos, and J. W. Rector III. High-resolution crosswell imaging of a west texas carbonate reservoir: Part I-project summary and interpretation. Geophysics, 60:667-681, 1995.

[19] C. Kao, S. Osher, and J. Qian. Legendre transform based fast sweeping methods for static Hamilton-Jacobi equations on triangulated meshes. J. Comput. Phys., 227:10209-10225, 2008.

[20] C. Y. Kao, S. J. Osher, and J. Qian. Lax-Friedrichs sweeping schemes for static HamiltonJacobi equations. J. Comp. Phys., 196:367-391, 2004.

[21] Y. Kurley, M. Lassas, and G. Uhlmann. Rigidity of broken geodesics flow and inverse problems. Amer. J. Math., 132:529-562, 2010.

[22] S. Leung and J. Qian. An adjoint state method for three-dimensional transmission traveltime tomography using first-arrivals. Comm. Math. Sci., 4:249-266, 2006.

[23] S. Leung and J. Qian. Transmission traveltime tomography based on paraxial Liouville equations and level set formulations. Inverse Problems, 23:799-821, 2007.

[24] P. L. Lions. Generalized solutions of Hamilton-Jacobi equations. Pitman Advanced Publishing Program, 1982.

[25] Z. Liu and N. Bleistein. Migration velocity analysis: theory and an iterative algorithm. Geophysics, 60:142-153, 1995.

[26] S. Luo and J. Qian. Factored singularities and high-order lax-friedrichs sweeping schemes for point-source traveltimes and amplitudes. Preprint, xxx:xxx, 2010. 
[27] B. T. May and J. D. Covey. An inverse ray method for computing geologic structures from seismic reflections-Zero-offset case. Geophysics, 46:268-287, 1981.

[28] R. Michel. Sur la rigidite imposee par la longueur des geodesiques. (French) [on the rigidity imposed by the length of geodesics]. Invent. Math., 65:71-83, 1981.

[29] R. L. Nowack and C. Li. Application of autoregressive extrapolation to the cross-borehole tomography. Studia Geophysica et Geodaetica, 50:337-348, 2006.

[30] L. Pestov and G. Uhlmann. On characterization of the range and inversion formulas for the geodesic X-ray transform. Inter. Math. Res. Notices., 80:4331-4347, 2004.

[31] L. Pestov and G. Uhlmann. Two dimensional compact simple Riemannian manifolds are boundary distance rigid. Ann. Math., 161:1093-1110, 2005.

[32] P. Podvin and I. Lecomte. Finite difference computation of traveltimes in very contrasted velocity models: a massively parallel approach and its associated tools. Geophys. J. Int., 105:271-284, 1991.

[33] A. M. Popovici and J. A. Sethian. Three-dimensional traveltime computation using the fast marching method. In 67th Ann. Internat. Mtg., Soc. Expl. Geophys., Expanded Abstracts, pages 1778-1781. Soc. Expl. Geophys., Tulsa, OK., 1997.

[34] J. Qian and W. W. Symes. Adaptive finite difference method for traveltime and amplitude. Geophysics, 67:167-176, 2002.

[35] J. Qian and W. W. Symes. Finite-difference quasi-P traveltimes for anisotropic media. Geophysics, 67:147-155, 2002.

[36] J. Qian, Y. T. Zhang, and H. K. Zhao. Fast sweeping methods for eikonal equations on triangulated meshes. SIAM J. Numer. Analy., 45:83-107, 2007.

[37] J. Qian, Y. T. Zhang, and H. K. Zhao. Fast sweeping methods for static Hamilton-Jacobi equations on triangulated meshes. J. Sci. Comp., 31:237-271, 2007.

[38] F. Qin, Y. Luo, K. B. Olsen, W. Cai, and G. T. Schuster. Finite difference solution of the eikonal equation along expanding wavefronts. Geophysics, 57:478-487, 1992.

[39] V. G. Romanov. Inverse problems of mathematical physics. VNU Science Press BV, Utrecht, The Netherlands, 1987.

[40] W. A. Jr. Schneider, K. Ranzinger, A. Balch, and C. Kruse. A dynamic programming approach to first arrival traveltime computation in media with arbitrarily distributed velocities. Geophysics, 57:39-50, 1992.

[41] A. Sei and W. W. Symes. Gradient calculation of the traveltime cost function without ray tracing. In 65th Ann. Internat. Mtg., Soc. Expl. Geophys., Expanded Abstracts, pages 13511354. Soc. Expl. Geophys., Tulsa, OK, 1994. 
[42] A. Sei and W. W. Symes. Convergent finite-difference traveltime gradient for tomography. In 66th Ann. Internat. Mtg., Soc. Expl. Geophys., Expanded Abstracts, pages 1258-1261. Soc. Expl. Geophys., Tulsa, OK, 1995.

[43] V. A. Sharafutdinov. Integral geometry of tensor fields. VSP BV, Utrchet, The Netherlands, 1994.

[44] P. Stefanov and G. Uhlmann. Rigidity for metrics with the same lengths of geodesics. Math. Res. Lett., 5:83-96, 1998.

[45] P. Stefanov and G. Uhlmann. Stability estimates for the X-ray transform of tensor fields and boundary rigidity. Duke Math. J., 123(3):445-467, 2004.

[46] P. Stefanov and G. Uhlmann. Boundary rigidity and stability for generic simple metrics. J. Amer. Math. Soc., 18(4):975-1003, 2005.

[47] P. Stefanov and G. Uhlmann. Boundary rigidity and stability for generic simple metrics. J. Amer. Math. Soc., 18:975-1003, 2005.

[48] P. Stefanov and G. Uhlmann. Recent progress on the boundary rigidity problem. Electron. Res. Announc. Amer. Math. Soc., 11:64-70 (electronic), 2005.

[49] P. Stefanov and G. Uhlmann. Recent progress on the boundary rigidity problem. Electron. Res. Announc. Amer. Math. Soc., 11:64-70, 2005.

[50] P. Stefanov and G. Uhlmann. Integral geometry of tensor fields on a class of non-simple Riemannian manifolds. Amer. J. Math., 130(1):239-268, 2008.

[51] P. Stefanov and G. Uhlmann. Local lens rigidity with incomplete data for a class of non-simple Riemannian manifolds. J. Differential Geom., 82(2):383-409, 2009.

[52] P. Stefanov and G. Uhlmann. The geodesic X-ray transform with fold caustics. preprint, 2010.

[53] C. H. Sword. Tomographic determination of interval velocities from reflection seismic data: the method of controlled directional reception. PhD thesis, Stanford University, Stanford, CA94305, 1987.

[54] C. Taillandier, M. Noble, and H. Calandra. A massively parallel 3-D refraction traveltime tomography algorithm. In 70th EAGE Conference $\&$ Exhibition, pages 1-5. EAGE, 2008.

[55] C. Taillandier, M. Noble, H. Chauris, P. Podvin, H. Calandra, and J. Guilbot. Refraction traveltime tomography based on adjoint state techniques. In 69th EAGE Conference $\mathcal{E}$ Exhibition, pages 1-5. EAGE, 2007.

[56] R. Tsai, L.-T. Cheng, S. J. Osher, and H. K. Zhao. Fast sweeping method for a class of Hamilton-Jacobi equations. SIAM J. Numer. Analy., 41:673-694, 2003.

[57] J. van Trier and W. W. Symes. Upwind finite-difference calculations of traveltimes. Geophysics, $56: 812-821,1991$. 
[58] J. Vidale. Finite-difference calculation of travel times. Bull. Seis. Soc. Am., 78:2062-2076, 1988.

[59] J. K. Washbourne, J. W. Rector, and K. P. Bube. Crosswell traveltime tomography in three dimensions. Geophysics, 67:853-871, 2002.

[60] C. A. Zelt and P. J. Barton. Three-dimensional seismic refraction tomography: a comparison of two methods applied to data from the Faeroe Basin. J. Geophys. Res., 103:7187-7210, 1998.

[61] H. K. Zhao. Fast sweeping method for eikonal equations. Math. Comp., 74:603-627, 2005. 